\title{
Role of negatively charged defects in the lattice contraction of Al-Si-N
}

\author{
C. A. Pignedoli, ${ }^{\text {a) }}$ D. Passerone, H. J. Hug, A. Pélisson-Schecker, and J. Patscheider \\ Swiss Federal Laboratories for Materials Testing and Research, Empa, Dübendorf, \\ Zuerich 8600, Switzerland
}

(Received 14 November 2009; accepted 22 January 2010; published online 17 February 2010)

\begin{abstract}
Experiments reveal that incorporation of substitutional Si in wurtzite AIN up to 6 at. \% results in a lattice contraction in the [0001] direction. The contraction is linear and, for higher silicon contents, the lattice parameters remain constant. We investigate the geometric and electronic properties of $\mathrm{Al}-\mathrm{Si}-\mathrm{N}$ compounds with $\mathrm{Si}$ content varying from 0 to 9 at. $\%$ by means of $a b$ initio simulations based on density functional theory. We demonstrate that charged defects are necessary to support the experimental evidence of a shrinking cell parameter: an ideal $\mathrm{Al}-\mathrm{Si}-\mathrm{N}$ wurtzite structure with delocalized charges would undergo lattice expansion due to Coulomb repulsion upon $\mathrm{Si}$ incorporation. Charged defects that act as acceptors and compensate the excess charge coming from $\mathrm{Si}$ overcompensate the lattice expansion and therefore promote a lattice contraction. (C) 2010 American Institute of Physics. [doi:10.1063/1.3323093]
\end{abstract}

According to the only existing $\mathrm{Al}-\mathrm{Si}-\mathrm{N}$ ternary phase diagram, ${ }^{1} \mathrm{AlN}$ and $\mathrm{Si}_{3} \mathrm{~N}_{4}$ are fully immiscible. Experimental data $^{2-6}$ however revealed that, up to a few atomic percent (at. \%), Si can be incorporated in the wurtzite lattice and a linear decrease of the lattice parameters, and hence of the average bond length, is observed. ${ }^{3,5}$ It was concluded that a solid solution of $\mathrm{Al}_{1-x} \mathrm{Si}_{x} \mathrm{~N}(0 \leq x \leq 0.08)$ (Ref. 3) or 0.12 (Ref. 5)] is formed by substitutional incorporation of $\mathrm{Si}$ atoms on $\mathrm{Al}$ sites in AlN. Above 6 at. \% of Si (i.e., $x=0.12$ ), we found that the $c$ lattice parameter remained constant, showing that at such concentrations $\mathrm{Si}$ atoms are no longer incorporated into the AlN lattice. ${ }^{5}$ Instead, they segregate at the boundaries of $\mathrm{Al}_{0.88} \mathrm{Si}_{0.12} \mathrm{~N}$ crystallites, forming a silicon nitride layer.

Two questions arise from these experimental results. First, the origin of the lattice contraction in $\mathrm{Al}_{1-x} \mathrm{Si}_{x} \mathrm{~N}$ is not yet fully understood. The introduction of $\mathrm{Si}$ in AlN must be accompanied by the formation of charge compensation defects, but the role of such defects on the lattice volume was never studied. Second, the invariance of our coatings on substrate temperature during reactive sputtering deposition (200 and $\left.500{ }^{\circ} \mathrm{C}\right)$ and annealing $\left(1000{ }^{\circ} \mathrm{C}\right)$ seems to suggest that thermodynamically distinct phases are formed for the compositional regions above and below 6 at. \% Si. Unfortunately, the thermodynamics of the $\mathrm{Al}-\mathrm{Si}-\mathrm{N}$ systems remains largely unknown.

Several $a b$ initio investigations for neutral and charged defects in III-V semiconductors in the literature ${ }^{7}$ examine $\mathrm{Si}$ incorporation in AlN interstitial sites, in substitutional $\mathrm{N}$ $\left(\mathrm{Si}_{\mathrm{N}}\right)$ and $\mathrm{Al}\left(\mathrm{Si}_{\mathrm{Al}}\right)$ sites, and $\mathrm{DX}$ centers, a particular case where a substitutional $\mathrm{Si}$ leaves the original $\mathrm{Al}$ site and breaks one of its $\mathrm{Si}-\mathrm{N}$ bonds. The most stable geometry for $\mathrm{Si}$ incorporation either in a formal $1^{+}$charge state $^{8}$ or in a neutral state is reported to be $\mathrm{Si}_{\mathrm{Al}}$ (Ref. 9) seemingly in agreement with the reported experimental evidences of the formation of a substitutional $\mathrm{Al}_{1-x} \mathrm{Si}_{x} \mathrm{~N}$ solid solution. The available $a b$ initio studies, however, do not take into account the relaxation of the cell parameters and obtain different charge states by adding or removing electrons from the simu-

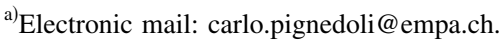

lation cell and including a uniform background charge to recover neutrality. The Si concentration of interest for the hard coating applications and investigated in our work is extremely high (few atomic percent) compared to the typical concentration of donors/acceptors in doped semiconductors. In the AlN-system the oxidation states of the $\mathrm{Al}$ and $\mathrm{N}$ are $3^{+}$ and $3^{-}$, respectively. Si has a $4^{+}$oxidation state. Hence, the replacement of an $\mathrm{Al}$ atom by a $\mathrm{Si}$ atom leads to a spatially increased charge density, since only three of the four electrons can be accepted by the N. This facilitates the formation of defects for formal-charge compensation. In the present study, we do not introduce artificial charges and compensation through uniform background, different combinations of point defects are introduced in the simulation cell and the cell parameters are allowed to relax. An aluminum vacancy $\left(V_{\mathrm{Al}}\right)$ is a common acceptor defect whose stability in the formal $3^{-}$state has been assessed theoretically (see, e.g., Ref. 10). A real system with $\mathrm{Si}$ in a formal $1^{+}$charge state can thus be represented by a simulation cell with three $\mathrm{Si}_{\mathrm{Al}}$ added for each $V_{\mathrm{Al}}$, with the latter formally charging itself with the three electrons coming from $\mathrm{Si}$ impurities. The ratio $1 V_{\mathrm{Al}} / 3 \mathrm{Si}_{\mathrm{Al}}$ corresponds to perfect stoichiometry in the mixing of $\mathrm{AlN}+\mathrm{Si}_{3} \mathrm{~N}_{4}$. Different charge states are represented by a $\mathrm{Si}_{\mathrm{Al}} / V_{\mathrm{Al}}$ ratio different from 3 and the neutral $\mathrm{Si}_{\mathrm{Al}}$ state requires no removal of $\mathrm{Al}$ atoms. An alternative compensation mechanism could be a DX center. DX1 (where a Si atom relaxes along the [0001] direction and breaks the corresponding bond to the $\mathrm{N}$ atom; see, e.g., Refs. 9 and 11 for a structural description of DX1 and DX2 defects) and DX2 (where one of the non-[0001] Si-N bonds is broken) are found to be the most stable configurations in a formally negative state. Since our case deals with a high density of Si impurities, it is important to note that the inclusion of two $\mathrm{Si}$ atoms in the same cell could account for both a negatively charged DX center (i.e., playing the role of the acceptor) and a $\mathrm{Si}_{\mathrm{Al}}$ (playing the role of the donor) in the formal $1^{+}$charge state. We investigated this additional compensation possibility. The present study, based on density functional theory, investigates at the atomistic level some of the mechanism described above that could lead to the observed behavior. In particular, the issue of lattice contraction will be addressed quantita- 


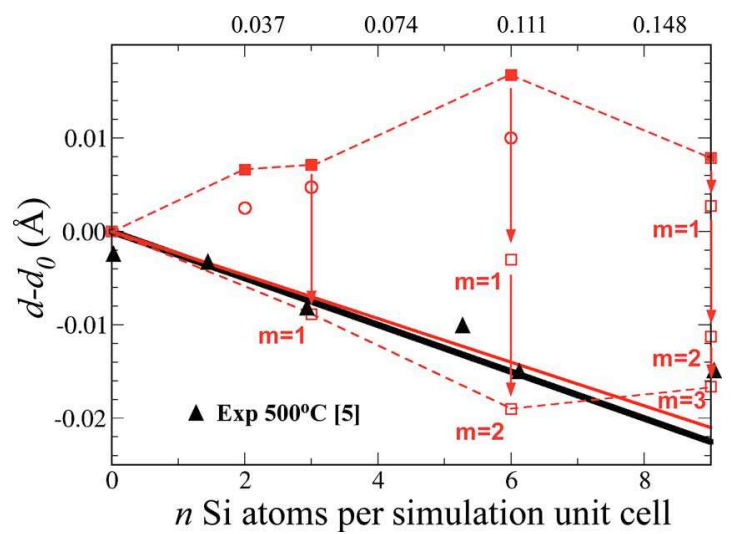

FIG. 1. (Color online) The fat solid and thin solid lines show the linear interpolation of $d-d_{0}$ with respect to experimentally (black) and theoretically (gray) determined bond lengths in wurtzite AlN and $\beta-\mathrm{Si}_{3} \mathrm{~N}_{4}$. Solid triangles are experimental data from Ref. 5 . The filled square points show the increase of the lattice parameter $(c=8 / 3 d)$ for $n=2,3,6,9 \mathrm{Si}_{\mathrm{Al}}$ in the simulation cell. The dotted lines are guides to the eye. The open squares indicate the reduction of the lattice parameter occurring for each Si concentration if $m \mathrm{Al}$ vacancies are additionally present. The arrows serve as guides to the eye. The open circles indicate the evolution of $d-d_{0}$ for $1,2,3 \mathrm{DX} 2$ centers introduced in addition to the $n=2,3,6 \mathrm{Si}_{\mathrm{Al}}$.

tively, whereas the stability problem will be extensively discussed and left as perspective.

We perform ab initio simulations of an AlN bulk with $\mathrm{Si}$ content of $0,1.9,2.8,5.6$, and 8.3 at. \%. The atomic models are built up from supercells equivalent to $3 \times 3 \times 3$ elementary cells of the ideal wurtzite structure for a total of 108 atoms. In this way, upon addition of single impurities, we can reproduce low $\mathrm{Si}$ content down to 1 at. \%. For the calculations we used the code $\mathrm{CP} 2 \mathrm{~K} ;{ }^{12}$ density functional theory was employed within local density approximation, ${ }^{13}$ electrons were represented with a mixed Gaussian-Plane Wave basis set approach, ${ }^{14,15}$ and the ionic potential was represented via Goedecker pseudopotentials. ${ }^{13}$ In addition to pure AlN and $\mathrm{Si}_{3} \mathrm{~N}_{4}$, we built up several structures: starting from a cell containing 108 atoms in the ideal wurtzite positions, we introduce $n=2,3,6,9 \quad \mathrm{Si}_{\mathrm{Al}}$ atoms and $m$ $=0, \ldots, n / 3 V_{\mathrm{Al}}(m=1,2,3)$. Our models consider random uniform distributions of $\mathrm{Si}_{\mathrm{Al}}$ and $V_{\mathrm{Al}}$. The atomic positions and lattice parameters of the simulation cell are then fully optimized. ${ }^{16}$

As shown in, ${ }^{5}$ the experimentally observed lattice contraction up to a silicon content of $x=0.12$ corresponds to a weighted average between the experimentally determined $d=1.8672 \AA(d=c 3 / 8)$ for pure wurtzite AlN and $d$ $=1.732 \AA$ for pure $\beta-\mathrm{Si}_{3} \mathrm{~N}_{4}$ (see solid fat line in Fig. 1). Our calculation gives slightly different values, namely 1.8515 and $1.725 \AA$ for the pure substances. The corresponding linear variation is shown by the thin solid red line in Fig. 1. The first important result of our calculation is that a $n=2,3,6,9$ substitution of the $\mathrm{Al}$ by $\mathrm{Si}$ leads to an increasing lattice parameter (see filled square points in Fig. 1), in contrast to our experimental observations. The additional charge introduced by silicon results in coulomb repulsion leading to an expansion of the lattice. In the following, we will therefore focus on models that contain at least one acceptor defect. In this paper we will consider Al vacancies and DX centers, leaving additional impurities like $\mathrm{O}$ and $\mathrm{H}$ atoms (in principle a realistic possibility) for a future work.

1) Compensation through Al vacancies. In Fig. 1 we

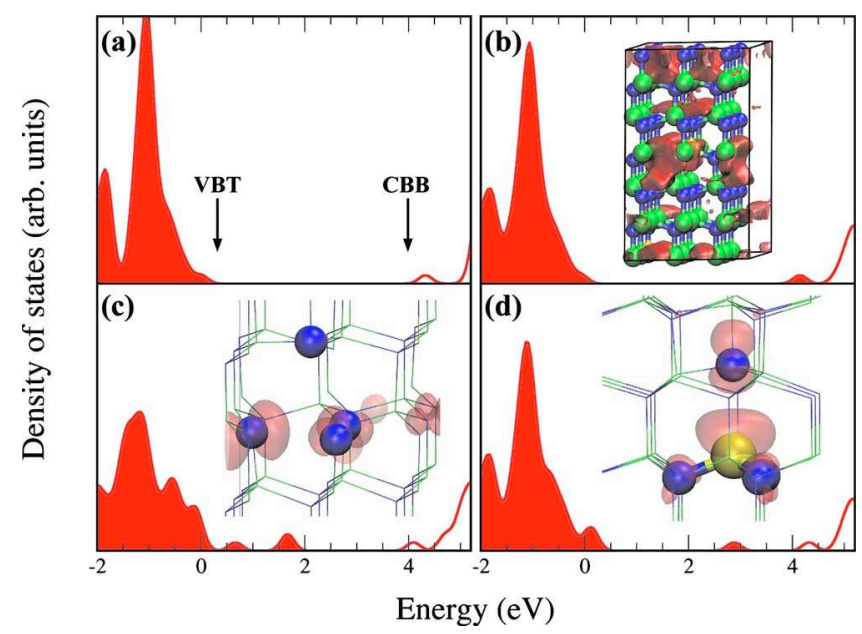

FIG. 2. (Color online) DOS close to VBT and CBB (indicated by arrows). The 0 is set to $E_{f}(A l N)$. Shaded/empty areas correspond to filled/empty states. (a) pure AlN. (b) $2 \mathrm{Si}_{\mathrm{Al}}$ in a supercell of 108 atoms; the peak close to CBB is now filled and corresponds to delocalized unpaired electrons. (c) 3 $\mathrm{Si}_{\mathrm{Al}}$ and $1 V_{\mathrm{Al}}$. Charge is localized in the $V_{\mathrm{Al}}$ and a state in the gap at $1.6 \mathrm{eV}$ is filled. (d) $2 \mathrm{Si}_{\mathrm{Al}}$ and $1 \mathrm{DX} 1$ center. Charge is in part localized on the DX1 and a state in the gap appears at $2.9 \mathrm{eV}$. The insets show the isosurface (transparent) of the charge density (value $0.007 \mathrm{e} / \AA^{3}$ ) for the highest occupied state superimposed to the atomic structure (dark: $\mathrm{N}$; gray: $\mathrm{Al}$; light gray: $\mathrm{Si}$ ) in the three nonpure systems. The ability of defects to localize charge is evident in the difference between (b) and (c and d).

show the bond lengths $d$, calculated from the $c$ lattice parameters as $d=3 / 8 c$, obtained for our structures. As shown in Fig. 1, the introduction of $V_{\mathrm{Al}}$ leads to a lattice contraction. For $n=3$ we find a $d$-value slightly below the experimentally observed one (see open square $m=1$ for $n=3$ in Fig. 1.). At $n=6$, one and two $V_{\mathrm{Al}}$ again lead to a lattice contraction (see arrow between filled and open squares). For $m=2$, the calculated $d$-value is again slightly below the experimentally observed value. At $n=9$ contraction deviates from linearity in agreement with experiments. Agreement with experiment at this concentration could be incidental since no phase segregation (that may occur above $n=6$ ) can be reproduced within our simulations; for this reason regions of higher $\mathrm{Si}$ concentrations were not investigated in the present study. In a real system we cannot expect a stoichiometric distribution of $V_{\mathrm{Al}}$. Regions of low $V_{\mathrm{Al}}$ concentration (corresponding to a lattice expansion) and of high $V_{\mathrm{Al}}$ concentration (leading to a lattice contraction, possibly larger than that predicted by linearity) may, however, coexist, leading to the experimentally observed overall lattice contraction. In addition, a combination of $V_{\mathrm{Al}}$ with other defects (e.g., DX centers) would still explain the experimentally observed lattice contraction. To understand qualitatively the effect of $V_{\mathrm{Al}}$ on the electronic properties of the alloy we can refer to Fig. 2: Figure 2(a) sketches the DOS of pure AlN. Addition of $\mathrm{Si}_{\mathrm{Al}}$ without compensating defects results in population of the conduction band bottom (CBB) and in delocalized charge [see Fig. 2(b) and inset] responsible for Coulomb repulsion. If $V_{\mathrm{Al}}$ is added, an electronic state is populated in the AlN gap close to the valence band top (VBT) and the charge is partly localized on $V_{\mathrm{Al}}$ [Fig. 2(c) and inset].

2) Compensation through DX centers. We investigated the effect of DX centers in structures with $n=2,3$, and $6 \mathrm{Si}$ atoms. In all cases introduction of DX centers results in partial localization of the Si unpaired electrons in the defect and in the population of electronic states in the gap close to the 
VBT [see Fig. 2(d) and inset]. Introduction of DX centers with a concentration higher than the ideal $n / 2$ stoichiometry was not possible in the case of $n=2$ and limited to $2 / 3 n$ in the case of $n=3$. Geometry optimization starting from a configuration with higher concentration of DX centers resulted in the removal of excess DXs. From our results it is evident that compensation through DX centers leads to a smaller lattice expansion, as compared to the case without compensation defects (compare round points to filled squared points in Fig. 1). However, only DX2 is able to limit the expansion of the c lattice parameter. Moreover, it is evident that DX2 alone (round points in Fig. 1) are not able to explain the lattice contraction revealed by the experiments. In conclusion we confirm that a combination of suitable densities of DX centers and $V_{\mathrm{Al}}$ can obviously match the experimental observations for all relevant concentrations of Si in AlN. In agreement with ${ }^{3}$ the a-lattice parameter is reduced upon $\mathrm{Si}$ incorporation and its variation is less evident with respect to the one of the c-lattice parameter. Variation of $a$ is mainly affected by $\mathrm{Si}_{\mathrm{Al}}$ rather than by $V_{\mathrm{Al}}$ or DX2 centers. The volume contractions or expansions are mainly related to the variation of the c-lattice parameter.

3) Thermodynamic considerations. A correct comparison of the energetics of the different structures considered in our investigation is not trivial. We distinguish between two cases: (a) For structures whose composition is stoichiometric with respect to $\mathrm{AlN}+\mathrm{Si}_{3} \mathrm{~N}_{4}$, a direct comparison of the energy of structure Al-Si-N with the separated $\mathrm{AlN}$ and $\mathrm{Si}_{3} \mathrm{~N}_{4}$ phases is correct. From our results it is evident that phase separation is always thermodynamically favored with respect to mixing in a wurtzite like structure. For $n=3$, phase segregation is favored by $0.16 \mathrm{eV}$ per wurtzite unit cell (four atoms). Phase segregation is dramatically favored upon increase of $n$, with an energy gain of 0.33 and $0.36 \mathrm{eV}$ for $n$ $=6,9$, respectively. (b) For structures that are nonstoichiometric with respect to $\mathrm{AlN}+\mathrm{Si}_{3} \mathrm{~N}_{4}$ the chemical potential of the $\mathrm{Al}, \mathrm{N}$, and $\mathrm{Si}$ atoms should be known to define the relative formation energy between two models as ${ }^{17}$

$$
\Delta G_{1-2}=E_{1}-E_{2}-\Delta N_{\mathrm{Si}} \mu_{\mathrm{Si}}-\Delta N_{\mathrm{Al}} \mu_{\mathrm{Al}}-\Delta N_{\mathrm{N}} \mu_{\mathrm{N}},
$$

where $E_{i}$ is the ab initio total energy of model $i, \Delta N_{x}$ is the difference between the numbers of atoms of species $x$ in structures 1 and 2, and $\mu_{x}$ is the chemical potential of species $x$. We consider equilibrium of $\mathrm{Al}$ and $\mathrm{N}$ with bulk AlN and the equilibrium of $\mathrm{Si}$ and $\mathrm{N}$ with $\mathrm{Si}_{3} \mathrm{~N}_{4}$ and discuss the tendency to segregate an $\mathrm{Al}$ atom in the different structures in order to recover stoichiometry with respect to $\mathrm{AlN}+\mathrm{Si}_{3} \mathrm{~N}_{4}$. If we assume $\mu_{\mathrm{Al}}+\mu_{\mathrm{N}}=E_{\mathrm{AlN}}$ (equilibrium with bulk AlN) and $3 \mu_{\mathrm{Si}}+4 \mu_{\mathrm{N}}=E_{\mathrm{Si}_{3} \mathrm{~N}_{4}}$ (equilibrium with bulk $\mathrm{Si}_{3} \mathrm{~N}_{4}$ ), we find that $\mu_{\mathrm{Al}}$ diminishes by $5 \mathrm{eV}$ going from $n=3, V_{\mathrm{Al}}=0$ to $n$ $=9, V_{\mathrm{Al}}=3$. This means that increasing the number of $\mathrm{Si}$ and $V_{\mathrm{Al}}$ the system becomes more and more unstable and phase segregation becomes more favorable.

The tendency to phase separation is not incompatible with the evidence of an Al-Si-N compound: the alloy is obtained in nonequilibrium conditions (namely sputter deposition at low temperatures) and the matrix of the dominant crystal (in this case wurtzite AlN) is driving the ordered arrangement of the atoms. As a comparison we can refer to recent works on complex compounds, e.g., Hafnium silicates. ${ }^{18}$ Hafnium silicates $\left(\mathrm{Hf}_{x} \mathrm{Si}_{1-x} \mathrm{O}_{2}\right)$ are thermodynamically not stable with respect to phase separation into
$\mathrm{HfO}_{2}$ (usually monoclinic structure with $\mathrm{Hf}$ sevenfold coordinated to $\mathrm{O}$ ) and $\mathrm{SiO}_{2}$ (quartz or crystobalite with $\mathrm{Si}$ fourfold coordinated to $\mathrm{O}$ ). However different stoichiometries of a stable compound can be formed even by molecular beam epitaxy. The reason for this is that at low $\mathrm{Hf}$ concentration the $\mathrm{SiO}_{2}$ matrix is able to accommodate $\mathrm{Hf}$ in a fourfold coordination, the energy loss paid for the unusual coordination of $\mathrm{Hf}$ is much lower than the one paid for the formation of defects that would be implied accommodating $\mathrm{Hf}$ in higher coordination. In our case, at low Si content the AlN matrix is the dominant environment that forces Si to occupy wurtzite sites. There is no crystalline form known to accommodate $\mathrm{Al}-\mathrm{Si}-\mathrm{N}$ at intermediate compositions, and for this reason only the low solubility limit can be realized: at high Si content the AlN matrix is not able anymore to preserve its symmetry and huge distortions toward a phase segregation will occur.

In conclusion, our $a b$ initio simulations give insight in the geometry and electronic properties of $\mathrm{Al}-\mathrm{Si}-\mathrm{N}$ compounds. We demonstrate that charged defects in the crystal must be present to explain the experimental evidence of lattice contraction upon $\mathrm{Si}$ incorporation. Due to Coulomb repulsion an ideal wurtzite Al-S-N compound undergoes expansion with increased $\mathrm{Si}$ content; the introduction of negatively charged DX centers limits this effect. The inclusion of aluminum vacancies in stoichiometric concentration (one $V_{\mathrm{Al}}$ each three $\mathrm{Si}$ ) results in a lattice reduction more marked with respect to measurements. We propose therefore that a combination of charged defects in proper concentration would explain the experimental curve. Although the formation of a Al-Si-N compound is thermodynamically unstable with respect to phase segregation, alloying in the wurtzite structure at low $\mathrm{Si}$ content is driven by the stable crystalline phase of the majority component.

We thank E. Tosatti for fruitful discussions and the Swiss National Supercomputing Center for computational resources.

${ }^{1}$ F. Weitzer, K. Remschnig, J. C. Schuster, and P. Rogl, J. Mater. Res. 5, 2152 (1990).

${ }^{2}$ M. Kasu, Y. Taniyasu, and N. Kobayashi, Jpn. J. Appl. Phys., Part 2 40, L1048 (2001).

${ }^{3}$ Y. Taniyasu, M. Kasu, and N. Kobayashi, Appl. Phys. Lett. 79, 4351 (2001).

${ }^{4}$ Y. Taniyasu, M. Kasu, and T. Makimoto, Nature (London) 441, 325 (2006).

${ }^{5}$ A. Pelisson, M. Parlinska-Wojtan, H. J. Hug, and J. Patscheider, Surf. Coat. Technol. 202, 884 (2007).

${ }^{6}$ A. Pelisson, Ph.D. thesis, University of Basal, Switzerland, 2009.

${ }^{7}$ C. Van de Walle and J. Neugebauer, J. Appl. Phys. 95, 3851 (2004).

${ }^{8}$ In chemistry, a formal charge is the hypothetical charge assigned to an atom.

${ }^{9}$ P. Bogusławski and J. Bernholc, Phys. Rev. B 56, 9496 (1997).

${ }^{10}$ C. Stampfl and C. G. Van de Walle, Phys. Rev. B 65, 155212 (2002).

${ }^{11}$ C. H. Park and D. J. Chadi, Appl. Phys. Lett. 66, 3167 (1995).

${ }^{12}$ J. VandeVondele, M. Krack, F. Mohamed, M. Parrinello, T. Chassaing, and J. Hutter, (2005), http://cp2k.berlios.de/.

${ }^{13}$ S. Goedecker, M. Teter, and J. Hutter, Phys. Rev. B 54, 1703 (1996).

${ }^{14}$ G. Lippert, J. Hutter, and M. Parrinello, Mol. Phys. 92, 477 (1997).

${ }^{15}$ G. Lippert, J. Hutter, and M. Parrinello, Theor. Chem. Acc. 103, 124 (1999).

${ }^{16}$ See supplementary material at http://dx.doi.org/10.1063/1.3323093 for the details of lattice parameters and cell volumes.

${ }^{17}$ W. Zhang, J. R. Smith, and X.-G. Wang, Phys. Rev. B 70, 024103 (2004).

${ }^{18}$ C. A. Pignedoli, A. Curioni, and W. Andreoni, Phys. Rev. Lett. 98 037602 (2007). 